\title{
Exploring Hong Kong Nursing Students' Experience of Using Smartphones in Clinical Practicum
}

\author{
Keung Sum Chan, Yee Shan Chan \\ School of Nursing, Tung Wah College, Hong Kong, China \\ Email: kschan@twc.edu.hk
}

How to cite this paper: Chan, K.S. and Chan, Y.S. (2021) Exploring Hong Kong Nursing Students' Experience of Using Smartphones in Clinical Practicum. Journal of Biosciences and Medicines, 9, 194-207. https://doi.org/10.4236/jbm.2021.96018

Received: May 16, 2021

Accepted: June 27, 2021

Published: June 30, 2021

\begin{abstract}
Background: The last three decades have seen a growing trend towards information, communication, and technology (ICT). People can access internet connection easily by a different electronic device for social or other purposes. There is considerable research on mobile learning which is getting more common in higher education for its effectiveness. The smartphone can allow the nursing student to access the internet for evidence-based knowledge in a handheld device at the point of care. Aim: This study aimed to explore the experience of nursing students while using the smartphone in the clinical practicum at the point of care. Study design: A qualitative descriptive approach was adopted. Methods: A total of 18 nursing students from three tertiary institutions participated in the semi-structured interview with simultaneously audio-taped and field-notes documented to explore their experience of using smartphones in the clinical settings at the point of care. Conventional content analysis was utilized to extract significant themes, categories, and subcategories. Results: Six key themes were identified including working environment, achieving information, feelings towards the use of smartphones, possible outcomes related to the use of smartphones in the ward setting, reactions towards negative outcomes related to use of smartphones in the ward setting, recommendations on facilitating the usage of the smartphone. Conclusion: Exploring the experience of Hong Kong nursing students in the present study indicated that the use of a smartphone could be a valuable resource that could contribute to a better learning outcome for the students in the clinical practicum.
\end{abstract}

\section{Keywords}

Mobile Learning, Smartphones, Nursing Students, Clinical Practicum, Hong Kong, Qualitative Research 


\section{Introduction}

In the past three decades, information and communication technology (ICT) has become an indispensable part of people's daily life, people can easily obtain information from the internet without any space and time limitations. According to the statistics by the Census and Statistics Department of the Hong Kong government (2021), almost 6 million persons had a smartphone in Hong Kong. The penetration rate of the smartphone being observed in persons aged 10 and over has increased from $54.0 \%$ to $92.1 \%$ during the period from 2012 to 2020 [1]. With the rapid development of mobile technologies and mobile devices, mobile learning has made great progress in nursing education in many countries [2] [3] [4] [5]. Nursing students take the privilege of the attributes of mobile learning with its portability and accessibility for granted and enable them to link the theoretical knowledge to the more complicated real-life situation which increases their self-confidence and learning efficiency [6] [7]. Numerous western studies explored the use and the positive influences of mobile learning in nursing clinical practicum; however, there are few studies about the experience of Hong Kong nursing students using smartphones in their clinical placement.

\section{Literature Review}

Considerable studies have provided an overview of the use of mobile technologies has many advantages on various clinical practice and educational outcomes in nursing education as it provides quick access for the students to verify their knowledge as well as tackle the difficulties in the clinical setting [6] [8] [9] [10] [11]. A certain mobile guiding system developed to train the students in the nursing clinical practice, students were supervised via the mobile devices to interact with simulated standard patients to facilitate them with specific skills to identify the abnormal symptoms from the tailor-made scenarios [12]. It indicated that the students using the mobile guiding system surpassed those using traditional ways in disease identification learning achievement [12].

For the learning outcomes of using smartphones in clinical practicum, numerous studies highlighted the theory-practice gap is a common problem for nursing students in the clinical practicum since it is difficult for students to match the theory and knowledge learned from the textbook to the clinical situation [13] [14]. Roberts and Williams (2017) indicated that mobile technology can narrow the gap as smartphones provide a shortcut and or as a referencing tool for the students to access the evidence-based information to reduce medical errors in the clinical practicum and also facilitate students' learning attitude and increase their interests as they can find out the answer immediately and think broader and more profound than getting the answer from the lecturers [15] [16] [17] [18]. Mobile learning not only brings positive feelings to the students but also improves their practical skills since it is a self-directed learning method, nursing students can access useful information repeatedly and practise their skills anytime and anywhere as well as mproves the quality of patient care and 
patient safety [19] [20]. Concerning patients' privacy and security, some senior clinical staff or clinical tutors do not allow the students to use smartphones to search for information in the ward setting and even some students refuse to do it due to being perceived as unprofessional and incompetent [6] [7] as well as overusing and over-relying on the smartphone [21]. Further, some technological barriers such as the limited battery life and slow network also restrict the use of smartphones [22].

A previous study indicated that the characteristics of teaching methods are different between Asian and western countries due to its educational conditions, and culture [23]. Nursing education in western countries generally emphasizes the skill of critical thinking which encourages the students to gather and seek information from a wide range of sources instead of accepting the information from others [24] [25] [26] whereas Hong Kong nursing students think less critically than western nursing students [27]. Many studies have well explored the use of PDA among healthcare professions in western countries [28] [29]. The different learning methods, educational conditions and culture among Asians and western contribute to our research topic.

\section{Research Gaps and Aim of the Study}

With the development and integration of mobile technology, smartphones have a high penetration than PDA and better suit for nursing education but the impacts of using smartphones among nrsing students in practicum is relatively unclear. Therefore, this study aimed to explore Hong Kong nursing students' experience of using smartphones in clinical practicum.

\section{Study Design}

A descriptive qualitative approach was employed.

\section{Setting and Recruitment}

This study was conducted to targeted undergraduate nursing students from different universities to achieve an in-depth understanding and experience of interviewees [30]. Convenience and snowball sampling strategies were used to recruit eligible nursing students for this study in mid-January 2018. For the inclusion criteria, students who could speak fluent Cantonese and able to give evidence of enrolling in the hospital clinical practicum at least two weeks also had the experience of using the smartphone with internet access during the practicum. To reduce the variation of the data, students who studied nursing programmes in other countries were excluded from this study. This research eventually recruited 18 participants and reached data saturation in which three consecutive participants did not share any new idea in the interview [31].

\section{Instrument}

A semi-structured interview guide was developed based on the literature review of the previous related English qualitative studies. A pilot study was conducted 
on 2 participants to test and modify the interview questions to establish the final one for subsequent interviews. The final version of the interview questions in the interview guide was as follows:

1) Describe when will you use the smartphone to search for evidencebased knowledge during clinical practicum?

2) In what situations did you use the smartphone to assist you in searching evidence-based knowledge during clinical practicum?

3) What are your thoughts on the use of smartphones in searching during nursing clinical practicum?

4) What obstacles have you faced when you tried to use a smartphone during clinical practicum?

5) Will you continue to use a smartphone in your nursing clinical practice while delivering care to patients?

6) Describe why do you use any other alternatives rather than using a smartphone to search for evidence-based knowledge?

7) What are your opinions on promoting the use of smartphones in searching evidence-based knowledge in clinical areas? What are your recommendations?

\section{Data Collection Method}

Face to face and semi-structured interview was conducted in the meeting room of the teaching college from the mid-January to April 2018. All interviews were used in Cantonese and audio-taped. Each interview lasted for approximately 30 to 45 minutes.

\section{Data Analysis}

All audiotaped interviews were transcribed verbatim by a researcher at the beginning of data analysis, as the researchers were native speakers so that another researcher was responsible for verifying the accuracy and correcting the errors in the transcription.

The qualitative content analysis technique mentioned by Graneheim and Lundman (2004) was employed to generate and integrate the results. The data analysis process was separated into two parts [32].

The first part was theme development from the transcription. Firstly, a researcher read through the transcripts several times to gain the core meaning of participants' ideas. Then the words, phrases about the students' experience of using smartphones were extracted and condensed into meaning units. These units were labeled with codes. After coding the whole transcript, those codes were compared to the differences and similarities, the codes that share a commonality were grouped into a category and subcategory. The recurring meaning together in the categories then formed a theme.

The second part was the translation of the established codes, categories, and themes. Two researchers translated the meaning units of perception from Chi- 
nese into English independently, another researcher then compared and interpreted these two English versions and generated a modified version. After that, the modified English version was back-translated into Chinese by another researcher. Researchers then compared the Chinese back-translated version to the original Chinese version to revise any inconsistencies. Researchers kept refining the modified English version till the English version could fully represent the original Chinese version. Member checking was done for validation.

\section{The Rigor of the Study}

Several strategies were employed to enhance the rigor of this study.

Member checking, that the participants were involved in the data analysis stage and provide a chance for them to read and make a comment on the finding, was employed to ensure the credibility and the dependability of this study [33].

To prolong the engagement, the length of the interview was extended to $30-60$ minutes. To facilitate the transferability, the information and the ideas of the participants were present in a narrative approach, some meaningful sentences in the conversation were directly quoted. Also, the description of the whole study was reported so that the readers could make a comparison to another study finding [33].

To ensure the stability or consistency of the inquiry processes, all the interviews were audio-taped, and processes and memos were written during the analyzing process for recording. The interview questions and probing questions were listed. The confirmability of this study was ensured as the progress and the detail of the study will be recorded. The question and the field notes would be the supplementary material for the audit trail as well so that readers could have a comprehensive understanding of this study.

\section{Ethical Consideration}

This study was approved by the Committee on the Use of Human and Animals Subjects in the teaching College. The researchers explained the details of the study to the volunteers. An information sheet was given to every participant who signed the written consent form before starting the interview. The participants had the right to withdraw from the study anytime without explaining their decision.

\section{Findings of the Qualitative Study}

\subsection{Description of the Participants}

Eighteen participants were recruited and there were 10 women and 8 men aged between 21 to 24 years old. Thirteen students came from Tung Wah College (TWC), two from the Hong Kong Polytechnic University (POLYU), two from the Chinese University of Hong Kong (CUHK), and one from the Hong Kong University (HKU). Fourteen participants were degree students and four from 
high diploma (HD). All of them had more than 8 weeks' clinical practicum experience. Keeping the anonymity, eighteen participants were assigned a code from A to R, respectively in the study.

\subsection{Qualitative Findings}

Six key themes were identified including working environment, achieving information, feelings towards the use of smartphones, possible outcomes related to the use of smartphones in the ward setting, reactions towards negative outcomes related to use of smartphones in the ward setting, recommendations on facilitating the usage of the smartphone. The themes, categories and subcategories are shown in Table 1.

\subsubsection{Theme 1: Working Environment}

This theme included two categories: supportive environment and non-supportive environment.

- Supportive environment

Most of the interviewees pointed out that the working environment in the clinical setting affected the willingness of using a smartphone. A majority stated that the encouragement and understanding from the junior staff made them more comfortable using a smartphone in the ward. A participant shared:

Table 1. Themes and categories and subcategories.

\begin{tabular}{|c|c|c|}
\hline Themes & Categories & Subcategories \\
\hline Working environment & $\begin{array}{l}\text { - Supportive environment } \\
\text { - Non-supportive environment }\end{array}$ & \\
\hline Achieving information & - Nature of information & $\begin{array}{l}\text { - The convenience of } \\
\text { using a smartphone to } \\
\text { access information } \\
\text { - Ponderous text-based } \\
\text { information } \\
\text { - Possible difficulties } \\
\text { - Application of } \\
\text { information } \\
\text { - Scope of information }\end{array}$ \\
\hline $\begin{array}{l}\text { Feelings towards the use of } \\
\text { smartphone }\end{array}$ & $\begin{array}{l}\text { - Positive Feeling } \\
\text { - Negative Feeling }\end{array}$ & \\
\hline $\begin{array}{l}\text { Possible outcomes related to the use } \\
\text { of smartphones in the ward setting }\end{array}$ & $\begin{array}{l}\text { - Positive Outcome } \\
\text { - Negative Outcome }\end{array}$ & $\begin{array}{l}\text { - Better learning process } \\
\text { - Providing a better } \\
\text { quality of care to the } \\
\text { patient }\end{array}$ \\
\hline $\begin{array}{l}\text { Reactions towards negative outcomes } \\
\text { related to use of smartphone in the } \\
\text { ward setting }\end{array}$ & & \\
\hline $\begin{array}{l}\text { Recommendations on facilitating the } \\
\text { usage of smartphone }\end{array}$ & & \\
\hline
\end{tabular}


"Our ward staff keen on the nursing student to use more smartphone to search for information, like the staff as they will also use a smartphone to search for information. Actually...they prefer using smartphones and find for information together." (J: 25)

Non-supportive environment

Many participants described their experience of facing discouragement and misunderstanding from the senior staff and ward management about the use of smartphones in the ward setting. A participant expressed that:

"But for those more senior (senior staff), they may not encourage us to use smartphones.... Maybe somebody really takes photos secretly with the smartphone, so they would concern more about the patient privacy, not hoping any incident to happen, so do not hope them(students) to use the smartphone." (P: 16)

\subsubsection{Theme 2: Achieving Information}

The second theme was developed from the categories of accessibility of information and the nature of the information.

- Accessibility of information

Many participants said that they chose to use a smartphone instead of a textbook for searching information because of the approachability of the smartphone. These categories emerged from the sub-categories: (a) convenience of using a smartphone to access information, (b) ponderousness of text-based information, and (c) possible difficulties.

(a) The convenience of using a smartphone to access information

Some participants pointed out that using a smartphone was efficient and convenient. A participant reported:

"I think using a smartphone to search information much faster than searching in books, is more efficient." (C: 04)

(b) Ponderousness of text-based information

The reason for students using a smartphone because the textbook was not user-friendly. Participants shared that search information from the textbook was troublesome and time-consuming. A participant illustrated that:

“... you may not sure about the where are those leaflets located, if you have to read those leaflets, it will be troublesome and very time-consuming, meaning that you have to find it out and find the one I needed, actually it takes a lot of time." (L: 20)

(c) Possible difficulties

The majority adduced that the use of smartphones was be bound by some technical problems such as the low battery life and slow network speed. A participant said:

"Also, I believe it is about the network speed of the smartphone. If the 
speed of the smartphone is too slow, it will lose significance, loss the significance of being fast. For the third one, I believe it is the battery (of smartphone) problem, if we continuously find for information, I believe the battery will be used up and I can no longer search for information." (J: 35)

- Nature of information

Most of the participants claimed that the information gained from the smartphone was different from the textbook and some of them were also concerned that the application of that information. There were two subcategories included: (a) scope of information and (b) application of information.

(a) Scope of information

Participants said that there was a variety of information from the internet which was more detailed and up-to-date than the textbook. A participant stated:

"I think the smartphone is more up-to-date, because (the information) will keep on updating. Once the protocol is printed out, it may not update regularly maybe once a year or half a year to update them." (B: 39)

(b) Application of information

Most of the participants worried about the accuracy and the authenticity of the information on the internet. A participant explained that:

"I think some information may not be reliable, which means you may mistrust.... Maybe some drugs, it is used in Hong Kong but not in foreign countries...Foreign website may mention some drugs which are not widely used in hospitals in Hong Kong." (M: 27)

\subsubsection{Theme 3: Possible Outcomes Related to Use of Smartphone in the Ward Setting}

The third theme emerged from the following categories: positive outcome and negative outcome.

- Positive outcomes

Participants agreed that using smartphones in the clinical practicum brought positive influences to their learning process and improve their quality of care. There were two subcategories generated: (a) better learning process, (b) providing a better quality of care.

(a) Better learning process

Participants expressed that using smartphones help them merging clinical and academic learning together which gave them a deeper impression and increased their learning motivation. A participant stated:

"In the clinical setting, I can only learn about the clinical skill, but as the smartphone is emerging, it is a bridge to connect (knowledge) learning to clinical so that you can learn both knowledge and skill at the same time. You can learn clinical skills and knowledge together; you do not have to separate them." (F: 23)

(b) Providing better quality of care 
Participants also noted that the use of smartphones is beneficial for patient care as it can reduce the risk of harming patients and facilitate the process of providing care. Some participants expressed that:

“... if you do not know that he cannot be propped up... if you did not notice it, you will harm the case. But if you ... search it when you know you are not familiar with the case; the risk of committing error will be decreased." (H: 28) "There are different steps that I do not know about, I can also use the smartphone to search for the concrete procedure and how to perform it. Actually, it is helpful for me to take care of patients and on the academic aspect." (J: 20)

- Negative outcomes

A majority said that the use of smartphones led to a perceived lack of professionalism and misunderstanding by the patients and staff. Participants also expressed that it may lead to the violation of patient privacy. A participant said:

"Because firstly it will affect the professional image. Patients do not know if we are using the smartphone for searching information or using it for entertaining ourselves." (D: 13)

"...some staff do not support us to use a smartphone, they will think that students are taking photos of others, will it be related to the problem of privacy." (E: 03)

\subsubsection{Theme 4: Feelings towards the Use of Smartphone}

Participants shared two opposing feelings when they used smartphones for searching information in the ward. The two categories under this theme: Positive feelings and the negative feeling.

- Positive feelings

Some participants commented that using smartphone increase their self-esteem and confidence when performing the nursing procedure to the patients. A participant shared that:

“....Can answer louder and with more confidence when others raise questions. Certainly, not all information is applicable in the ward. But you will feel happy when you can gain extra knowledge, you think you can learn things. And the staff will think that you revised, and learned. You will not be unable to answer anything when you know nothing. I think it helps a lot towards students' self-confidence." (P: 14)

- Negative feelings

Although the use of smartphones can improve their satisfaction; however, some participants expressed that they were scolded for using a smartphone. Also, a majority felt worried and fear when using smartphones in front of the staff and patients. A participant stated:

“... He/she did not even come and take a look or figure out what we were 
doing, but directly blame us that we were playing smartphone. I think it is just like being accused wrongly." (E: 24)

\title{
10.2.5. Theme 5: Reaction towards Possible Outcomes Related to Use of Smartphone in the Ward Setting
}

Some participants took different actions when using smartphone in a non-supportive or uncertain clinical setting such as avoiding using smartphone in front of other, searching only when having free time. Some participants pointed out that self-discipline was important when nursing students using smartphones in the ward. Two participants mentioned that:

"Because when you face something that you do not know, you have to solve it, so I will keep away from staff, and go places where is no one there, like treatment room, to search information. This can solve the problem of being recognized and misunderstood by staff." (E: 64)

"If students are more self-discipline, knowing that they should only search for information to learn more, not replying WhatsApp. This is fine. If...using a smartphone is fine in the ward setting." (C: 28)

\subsubsection{Theme 6: Recommendations on Facilitating the Usage of Smartphone}

Some participants suggested that the Hospital Authority and the school had critical roles to facilitate the nursing students to use of smartphones in clinical practicum to improve their learning and caring of patients. A participant recommended that:

\begin{abstract}
"Maybe HA (hospital authority)... If you want to improve this (smartphone usage during practicum), I have mentioned that relatives do not like it... Promotion so that relatives will change their perception....about using the smartphone for a healthcare professional is a normal thing, you may have to do more promotion." (F: 36)
\end{abstract}

\section{Discussion}

The result of this study concurred with the current literature. Several previous studies have been shown that the environmental factor influences the nursing students' willingness of using smartphones in the clinical setting which corroborates our study [4] [34]. Most of the participants in this study identified the acceptance of senior and junior staff were different which affected their preferences of using smartphones to searching information. And majority pointed out that the variation of the staff was due to the acceptance of technology, stereotypes of smartphones, and the concern regarding patient privacy which were consistent with the past study [7].

The finding of possible outcomes and possible feelings also match with the finding reported in the current study [35] [36]. The majority shared that the use of smartphones facilitated their role of being a student and a healthcare provider since immediate searching information in the clinical setting helped them to 
bridge the theory-knowledge gap, they can merge the theoretical knowledge to the real situation. However, some participants expressed that worry about professionalism and perceived that staff and relatives presupposed they were leisure and not focus on their work. As the fear of being scold and misunderstanding, students resisted and declined the use of a smartphone as an educational device. In this study, several participants emphasized the opinion of looking professional in the eye of relatives which did not show in the previous study.

The finding of reaction and recommendation in this study were closer to the local situation and did not well illustrate in the previous studies. In the current study, some participants did not use the smartphone to search information when the ward environment was non-supportive, but a large group of participants voiced that they would either privately hide up in the treatment room and washroom in order to avoid being seen by staff and patients or only use smartphone after finishing all the work. Most of the participants stressed the importance of self-discipline which students should behave well and realize the role of being a healthcare professional so as to minimize the negative outcomes of using smartphones in the clinical practicum.

By promotion of mobile learning in the clinical setting, the misconception and misunderstanding could be rectified and cultivate the integration of using a smartphone. Moreover, the approval from the school made the participants feel more secured and encouraged them to learn more. The implementation of mobile learning in the hospital may need to concern different problems such as the privacy and trust between staff and students but the participants showed largely support the promotion.

\section{Conclusion}

To conclude, the qualitative descriptive study explored Hong Kong nursing students' experience towards using smartphones in the clinical practicum. The findings in the present study indicated that the use of a smartphone could be a valuable resource that could contribute to a better learning outcome for the students at the point of care. Also, the findings point up how culture might be a factor to affect their willingness and acceptance of using smartphones in a clinical setting which could be further explored.

\section{Acknowledgements}

We gratefully acknowledge the participants who have participated in this study. Without their participation, the study would not have been completed. We would like to thank our research team members' great contribution to the current study including Sui Sing CHEUNG, Nok Yiu FAN, Wai Tsam LAM, Yan Yi LAM, and Hoi Yung WONG.

\section{Conflicts of Interest}

The authors declare no conflicts of interest regarding the publication of this pa- 
per.

\section{References}

[1] Census and Statistics Department Hong Kong Special Administrative Region (2021) Thematic Household Survey Report-Report No. 73-Information Technology Usage and Penetration.

https://www.censtatd.gov.hk/en/data/stat report/product/B1130201/att/B113027320 21XXXXB0100.pdf

[2] Kukulska-Hulme, A. and Traxler, J. (Eds.) (2005) Mobile Learning: A Handbook for Educators and Trainers. Psychology Press.

[3] Dennison, L., et al. (2013) Opportunities and Challenges for Smartphone Applications in Supporting Health Behavior Change: Qualitative Study. Journal of Medical Internet Research, 15, E86. https://doi.org/10.2196/jmir.2583

[4] Beauregard, P., Arnaert, A. and Ponzoni, N. (2017) Nursing Students' Perceptions of Using Smartphones in the Community Practicum: A Qualitative Study. Nurse Education Today, 53, 1-6. https://doi.org/10.1016/j.nedt.2017.03.002

[5] Chang, C.Y., et al. (2018) Trends and Research Issues of Mobile Learning Studies in Nursing Education: A Review of Academic Publication from 1971 to 2016. Computers \& Education, 116, 28-48. https://doi.org/10.1016/j.compedu.2017.09.001

[6] O'Connor, S. and Andrews, T. (2016) Nursing Students' Opinion on the Use of Smartphone Applications (Apps) in Clinical Education and Training: A Study Protocol. Studies in Health Technology and Informatics, 225, 1024-1025.

[7] McNally, G., et al. (2017) Nurse Manager and Student Nurse Perceptions of the Use of Personal Smartphones or Tablets and the Adjunct Applications, as an Educational Tool in Clinical Settings. Nurse Education in Practice, 23, 1-7. https://doi.org/10.1016/j.nepr.2016.12.004

[8] Jeong, H. (2017) Effects of Nursing Students' Practices Using Smartphone Videos on Fundamental Nursing Skills, Self-Efficacy, and Learning Satisfaction in South Korea. Eurasia Journal of Mathematics, Science and Technology Education, 13, 2351-2365. https://doi.org/10.12973/eurasia.2017.01229a

[9] Church, K., et al. (2007) Mobile Information Access: A Study of Emerging Search Behavior on the Mobile Internet. ACM Transactions on the Web (TWEB), 1. https://doi.org/10.1145/1232722.1232726

[10] Phillippi, J.C. and Wyatt, T.H. (2011) Smartphones in Nursing Education. CIN: Computers, Informatics, Nursing, 29, 449-454. https://doi.org/10.1097/NCN.0b013e3181fc411f

[11] Guo, P., et al. (2016) An Integrative Review of the Impact of Mobile Technologies Used by Healthcare Professionals to Support Education and Practice. Nursing Open, 3, 66-78. https://doi.org/10.1002/nop2.37

[12] Wu, P.H., et al. (2011) A Pilot Study on Conducting Mobile Learning Activities for Clinical Nursing Courses Based on the Repertory Grid Approach. Nurse Education Today, 31, e8-e15. https://doi.org/10.1016/j.nedt.2010.12.001

[13] Landers, M.G. (2000) The Theory-Practice Gap in Nursing: The Role of the Nurse Teacher. Journal of Advanced Nursing, 32, 1550-1556. https://doi.org/10.1046/j.1365-2648.2000.01605.x

[14] Ajani, K. and Moez, S. (2011) Gap between Knowledge and Practice in Nursing. Procedia-Social and Behavioral Sciences, 15, 3927-3931.

https://doi.org/10.1016/j.sbspro.2011.04.396 
[15] Roberts, D. and Williams, A. (2017) The Potential of Mobile Technology (\# MoTech) to Close the Theory Practice Gap. Nurse Education Today, 53, 26-28. https://doi.org/10.1016/j.nedt.2017.03.003

[16] Kenny, R.F., et al. (2008) The Feasibility of Using Mobile Devices in Nursing Practice Education. https://doi.org/10.19173/irrodl.v10i3.730

[17] Kuiper, R. (2008) Use of Personal Digital Assistants to Support Clinical Reasoning in Undergraduate Baccalaureate Nursing Students. Comput. Inform. Nurs., 26, 90-98. https://doi.org/10.1097/01.NCN.0000304776.40531.bc

[18] Mackay, B.J., Anderson, J. and Harding, T. (2017) Mobile Technology in Clinical Teaching. Nurse Education in Practice, 22, 1-6. https://doi.org/10.1016/j.nepr.2016.11.001

[19] Kim, S.J., et al. (2017) A Smartphone Application to Educate Undergraduate Nursing Students about Providing Care for Infant Airway Obstruction. Nurse Education Today, 48, 145-152. https://doi.org/10.1016/j.nedt.2016.10.006

[20] Koren, A.L. and Fisher, K. (2007) Palm Perspectives: The Use of Personal Digital Assistants in Nursing Clinical Education. A Qualitative Study. Online Journal of Nursing Informatics, 11.

[21] Cho, S. and Lee, E. (2016) Distraction by Smartphone Use during Clinical Practice and Opinions about Smartphone Restriction Policies: A Cross-Sectional Descriptive Study of Nursing Students. Nurse Education Today, 40, 128-133.

https://doi.org/10.1016/j.nedt.2016.02.021

[22] Wu, C.C. and Lai, C.Y. (2009) Wireless Handhelds to Support Clinical Nursing Practicum. Journal of Educational Technology \& Society, 12.

[23] Lu, J., et al. (2016) Current Situation of the Learning Style of Chinese Medical Undergraduates and Its Consideration. International Journal of Clinical and Experimental Medicine, 9, 4243-4252.

[24] Wong, J.K.K. (2004) Are the Learning Styles of Asian International Students Culturally or Contextually Based? International Education Journal, 4, 154-166.

[25] Deng, F.F. (2015) Comparison of Nursing Education among Different Countries. Chinese Nursing Research, 2, 96-98. https://doi.org/10.1016/j.cnre.2015.11.001

[26] Kawashima, A. and Petrini, M.A. (2004) Study of Critical Thinking Skills in Nursing Students and Nurses in Japan. Nurse Education Today, 24, 286-292. https://doi.org/10.1016/j.nedt.2004.02.001

[27] Tiwari, A., Avery, A. and Lai, P. (2003) Critical Thinking Disposition of Hong Kong Chinese and Australian Nursing Students. Journal of Advanced Nursing, 44, 298-307. https://doi.org/10.1046/j.1365-2648.2003.02805.x

[28] Garrett, B.M. and Jackson, C. (2006) A Mobile Clinical e-Portfolio for Nursing and Medical Students, Using Wireless Personal Digital Assistants (PDAs). Nurse Education Today, 26, 647-654. https://doi.org/10.1016/j.nedt.2006.07.020

[29] While, A. and Dewsbury, G. (2011) Nursing and Information and Communication Technology (ICT): A Discussion of Trends and Future Directions. International Journal of Nursing Studies, 48, 1302-1310. https://doi.org/10.1016/j.ijnurstu.2011.02.020

[30] Lambert, V.A. and Lambert, C.E. (2012) Qualitative Descriptive Research: An Acceptable Design. Pacific Rim International Journal of Nursing Research, 16, 255-256.

[31] Francis, J.J., et al. (2010) What Is an Adequate Sample Size? Operationalising Data Saturation for Theory-Based Interview Studies. Psychology and Health, 25, 12291245. https://doi.org/10.1080/08870440903194015 
[32] Graneheim, U.H. and Lundman, B. (2004) Qualitative Content Analysis in Nursing Research: Concepts, Procedures and Measures to Achieve Trustworthiness. Nurse Education Today, 24, 105-112. https://doi.org/10.1016/j.nedt.2003.10.001

[33] Curtin, M. and Fossey, E. (2007) Appraising the Trustworthiness of Qualitative Studies: Guidelines for Occupational Therapists. Australian Occupational Therapy Journal, 54, 88-94. https://doi.org/10.1111/j.1440-1630.2007.00661.x

[34] Chan, D.S. and Ip, W.Y. (2007) Perception of the Hospital Learning Environment: A Survey of Hong Kong Nursing Students. Nurse Education Today, 27, 677-684. https://doi.org/10.1016/j.nedt.2006.09.015

[35] Swan, B.A., et al. (2013) Evaluating Tablet Technology in an Undergraduate Nursing Program. Nursing Education Perspective, 34, 192-193. https://doi.org/10.1097/00024776-201305000-00012

[36] Wittmann-Price, R., Kennedy, L. and Godwin, C. (2012) Use of Personal Phones by Senior Nursing Students to Access Health Care Information during Clinical Education: Staff Nurses' and Students' Perceptions. Journal of Nursing Education, 51, 642-646. https://doi.org/10.3928/01484834-20120914-04 